\title{
HEALTH RISK MANAGEMENT IN KINDERGARTENS, SCHOOLS AND UNIVERSITIES, DURING COVID-19 PANDEMIC: A HEURISTIC FRAMEWORK
}

\author{
Latchezar P. Tomov \\ Chief Assistant Professor \\ Department of Computer Science \\ New Bulgarian University, Sofia, Bulgaria \\ E-mail: lptomov@nbu.bg \\ Dr. Tsvetelina V. Velikova \\ Assistant Professor \\ Department of Clinical Immunology \\ University Hospital Lozenetz \\ Sofia University St. Kliment Ohridski, Sofia, Bulgaria \\ E-mail: tsvelikova@medfac.mu-sofia.bg \\ Dr. Hristiana M. Batselova \\ Assistant Professor \\ Department of Epidemiology and Hygiene \\ University Hospital "Saint George" \\ Medical University, Plovdiv, Bulgaria \\ E-mail: dr_batselova@abv.bg
}

\begin{abstract}
The SARS-COV-2 pandemic, causing COVID-19 had a specific pattern of spread in Bulgaria during several waves from 15.06.2020 to 31.03.2021. The measures, which the health authorities took to prevent the spread in the kindergarten and schools, combined with several other factors, specific for Bulgaria made them the main driver of the pandemic. In this article we propose heuristic framework as a strategy for health risk management, based on the properties of the virus and the properties of children's immune system.
\end{abstract}

Keywords: SARS-COV-2, COVID-19, Children, Health Risk Management, Heuristic Framework.

\section{JEL Classification Codes: I18.}

\section{INTRODUCTION}

The coronavirus SARS-COV-2 virus has a number of features that have caused a pandemic and posed a challenge to health authorities around the world, who must manage the risk of its spread with all its consequences, in the short and long term. We define the main health risk as the number of children infected; a secondary risk is the number of hidden infections, not uncovered by testing. 


\section{IDENTIFICATION OF HEALTH RISKS}

There are two types or health risk causes - created by human behavior and by the characteristics of the pathogen. Risks, created by human behavior are due to lack of information. Unlike probability one cannot assume symmetry of risks due to lack of information, but has to assume that risks are inversely proportional to the amount of information people have. Risks are measured with expectation, not probability, which is probability times payoff and payoff is asymmetrical - the price of preventative actions against risks which do not come to realization is finite, while the price of the realized risks without prevention is potentially infinite (pandemics are existential treat to humanity) (Siegenfeld et al., 2020).

Risks, cause by lack of information are manifested by human behavior - delayed reactions to pandemic treats; improper measures taken (assuming for example symptomatic transmission as default while SARS-COV-2 relies on asymptomatic and presymptomatic transmission as main drivers), lack of compliance from the public without enforcement; lack of information who is infected leading directly to increased prevalence and mortality; lack of information for long term health effects for the younger that leads directly to more risky behavior; lack of basic knowledge in biology and critical thinking that leads to vaccine skepticism (Kault, 2020). Government actions, related to concealing the actual mortality directly increase the pandemic risk, prevalence and mortality from the disease.

Risks caused by the virus itself - superspreading, asymptomatic transmission, aerosol transmission, long incubation period, long sickness and hospital treatment period, chemical stability of the virus, transmissibility, mutation rates (Kault, 2020).

- Superspreading creates risk of extremely high effective reproductive number before any measures are taken, interaction analogous to unstable nuclear division and atomic bombs for supercritical mass of Uranium-235 and describable with the same branching process.

- Asymptomatic transmission creates two types of risks - one is from the lack of information for the spread and thus lack of controllability of the pandemic, rendering other measures ineffective; the other is the health risk for asymptomatic individuals to develop sudden health deterioration due to lack of treatment and the presence of blood cloths that can lead to pulmonary embolism and death or long Covid issues. Not knowing who is sick due to lack of symptoms directly increases mortality.

- Aerosol transmission enables the superspreading of the virus and also makes all soft measures like 3 feet rules, helmets and hand washing ineffective. A single person infected 500 people in a temple in South Korea (and there are many more such cases), which is impossible to be done just by coughing and sneezing (Greenhalgh et al., 2021).

- The long incubation period decreases controllability of the pandemic, increases the pandemic duration and thus economic and psychological burden; it decreases confidence in the effect of pandemic measures due to the long delay between imposing them and the observed effect on the growth of cases.

- The long sickness period increases the risk of overburdening the healthcare system which leads to increased mortality.

- The chemical stability of the virus (1) (Fig. 1) decreases the seasonality of the pandemic and allows spreading in summer, which allows the virus to mutate and adapt to the selective pressure from the large number of people with naturally acquired immunity (Relman, 2020).

- The mutation rate of the virus although lower than the rates of influenza viruses is high enough to allow the appearance of multiple variants that evade natural immunity, thus 
prolonging the pandemic.

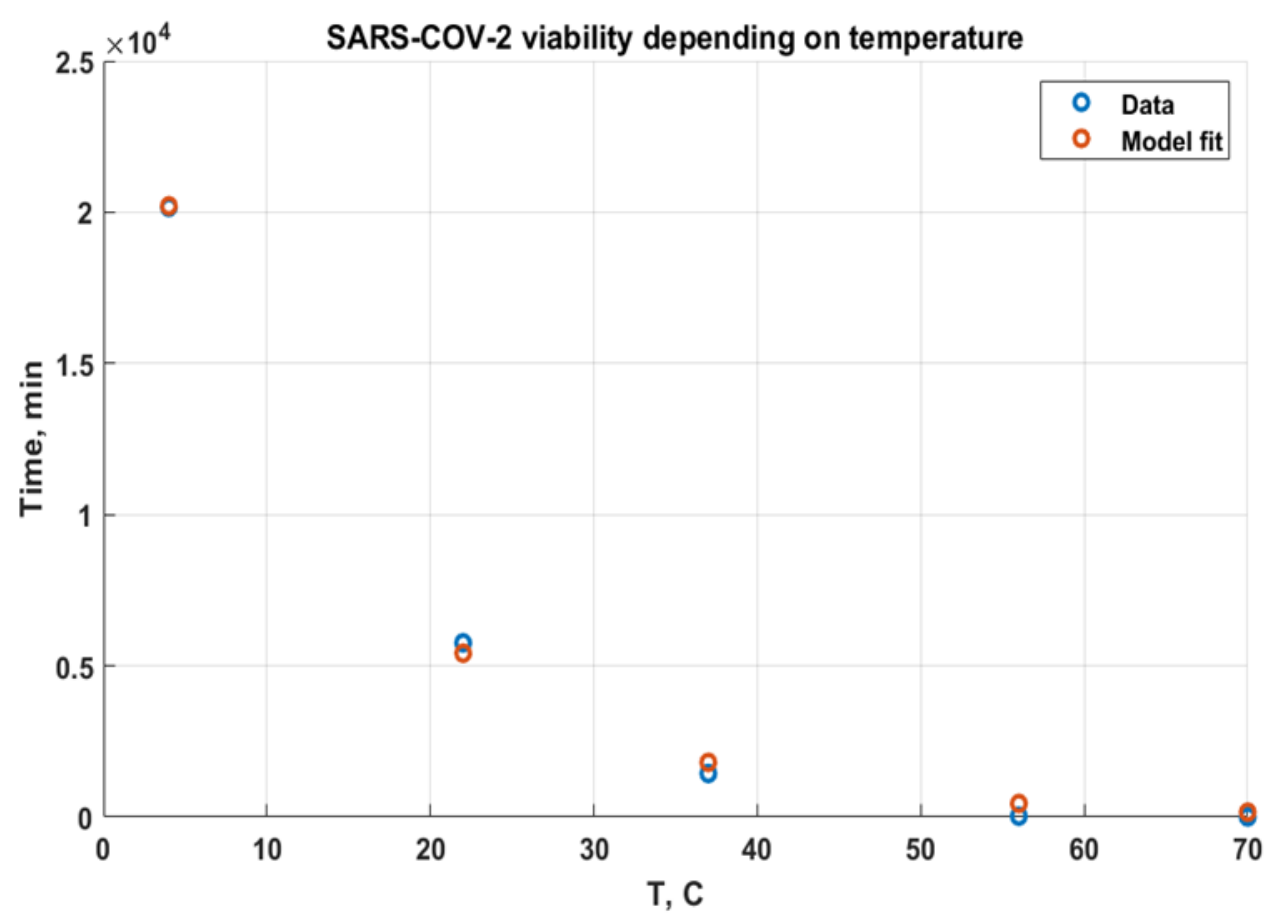

Figure 1. Exponential model for SARS-COV-2 viability as a function of temperature $t=a e^{b T}, a=27077.16941845914, b=-0.073146909350206$

\section{HEURISTIC FRAMEWORK FOR RISK MANAGEMENT}

All different characteristics impose different risks that require different measures. Superspreading events requires limiting of large gatherings to drive the effective reproductive number down - limiting the class size and the number of classes in schools as example. Asymptomatic transmission requires high frequency proactive testing and backwards contact tracing together with isolation for the infected (Fouzas, 2021). The high proportion of asymptomatic children and the lower antigen test sensitivity for asymptomatic cases together impose thee need to quarantine and isolate all contacts together with all members of the household. Masks, air ventilation and shorter duration of class hours is needed to battle aerosol transmission.

The complexity of the models needed to address with single point estimates all risks including behavioral ones is such, that in order the policy of risk management to be effective under rapidly changing circumstances, a much more flexible approach is needed. The heuristic framework we propose uses the ordinality of risks taken from qualitative assessment of epidemiological models to prioritize them and to propose a general strategy for imposing measures by addressing risks independently and simultaneously. Several different heuristic rules are proposed, together with an overall heuristic for the implementation of different policies that reflect them. We propose a subset of high priority risks - asymptomatic transmission, aerosol transmission and long incubation period for which complex measure are needed (superspreading needs only group size reduction). We give measurable parameters through which they are directly or indirectly expressed - number, duration and intensity of contacts. We assess which of the measurable parameters are controllable in kindergarten, school and university settings and 
which are not. We give an example of policy implementation, following the heuristic framework.

\section{CONCLUSION}

The novel coronavirus pandemic imposes unique challenges for containment due to several different pathogen characteristics and human behavior. Fairly complex epidemiological models failed to predict its development, or to control risk of spreading at educational facilities such as the University of Chicago [https://www.quantamagazine.org/the-hard-lessons-of-modeling-thecoronavirus-pandemic-20210128/]. The complexity of the combined pathogen and human behavior needs a flexible and adaptive approach, which for practical purposes is necessary to be heuristic. We propose such heuristic framework, tailored to educational facilities, which can easily be applied to other environments due to its agility and generality. Good judgement and the following of few simple rules is necessary in order to implement a successful strategy to control the spread of the virus.

\section{REFERENCES}

Fouzas, S. (2021). SARS-CoV-2 rapid antigen detection tests. The Lancet Infectious Diseases.

Greenhalgh, T., Jimenez, J. L., Prather, K. A., Tufekci, Z., Fisman, D., \& Schooley, R. (2021). Ten scientific reasons in support of airborne transmission of SARS-CoV-2. The lancet, 397(10285), 1603-1605.

Kault, D. (2020). Superspreaders, asymptomatics and COVID-19 elimination. Medical Journal of Australia, 213(10), 447-448.

Relman, D. A. (2020). Rapid Expert Consultation on SARS-CoV-2 Survival in Relation to Temperature and Humidity and Potential for Seasonality for the COVID-19 Pandemic.

Siegenfeld, A. F., Taleb, N. N., \& Bar-Yam, Y. (2020). Opinion: What models can and cannot tell us about COVID-19. Proceedings of the National Academy of Sciences, 117(28), 16092-16095.

\section{Copyrights}

Copyright for this article is retained by the author(s), with first publication rights granted to the journal. This is an open-access article distributed under the terms and conditions of the Creative Commons Attribution license (http://creativecommons.org/licenses/by/4.0/). 